\title{
Cosmic Visions in Celestial Mechanics
}

\author{
Alessandra Celletti • Antonio Giorgilli • \\ Ettore Perozzi · Sylvio Ferraz-Mello
}

Published online: 26 May 2010

(C) Springer Science+Business Media B.V. 2010

Modern Celestial Mechanics gains new momentum as its methods and results enter more and more into different academic fields and are, in turn, influenced by them. This is exactly what happens now: perturbation theories are a continuous source of inspiration for novel applications in spaceflight dynamics, while planetary dynamics must keep pace with the rapidly expanding field of exoplanets as well as with more traditional yet unresolved problems concerning the Solar System. In the short time span of a few years, significant results have been obtained on the subject of the long-standing questions on the stability of the inner planets. New and classical problems related to periodic orbits and chaotic diffusion have been investigated under a new light, as mission design is increasingly entering the realm of the three-body problem for computing station keeping strategies and transfer trajectories.

This exciting situation of cross-breeding among different communities is likely to further increase in the future, fostered by the ambitious plans of the major space agencies. The newly announced NASA plans call for a robust scientific program of robotic solar system exploration and of space observatories/probes, where the recent advances of Celestial Mechanics can play a crucial role. The European Space Agency has just approved a suite of new scientific missions and started the Space Situational Awareness program, whose aim is to monitor the

\footnotetext{
A. Celletti $(\varangle)$

Universita' di Roma Tor Vergata, Rome, Italy

e-mail: celletti@mat.uniroma2.it
}

A. Giorgilli

Dipartimento di Matematica,

Università degli Studi di Milano, via Saldini 50, 20133 Milano, Italy

E. Perozzi

Space Academy Foundation,

Telespazio S.p.A., Via Tiburtina 965, 00156 Rome, Italy

S. Ferraz-Mello

Instituto de Astronomia Geofísica e Ciências Atmosféricas,

Universidade de São Paulo, São Paulo, Brazil 
environment and the associated threats of the space surrounding our planet: a deep knowledge of space debris and NEO dynamics is essential to this end.

The content of the current special issue reflects the interdisciplinary nature of Celestial Mechanics, ranging from theoretical results to the study of the dynamics of natural and artificial bodies. The special issue focuses on the hot topics of Celestial Mechanics, some of which have been already included in the strategic plans of the main spatial agencies, like the "Cosmic Visions" program by ESA or the future plans recently announced by the NASA administrator, Charles Bolden. Indeed, the content of this special issue reflects the cornerstones to which Celestial Mechanics is presently oriented: a theoretical understanding of the theory of chaos and of the stability of planetary systems, the analysis and the role of mean-motion resonances, the determination and mitigation strategies of space debris and NEOs, advanced methods in flight dynamics based on dynamical systems theory and the investigation of the dynamical behavior of extrasolar planetary systems.

Sylvio Ferraz-Mello thanks the organisers of CELMEC V, A. Celletti, A. Giorgilli, E. Perozzi, G. B. Valsecchi, for allowing him to invite the speakers to submit their original papers to Celestial Mechanics \& Dynamical Astronomy for this special issue. The guest editors Alessandra Celletti, Antonio Giorgilli and Ettore Perozzi acknowledge the sponsors of CELMEC V: Italian Society of Celestial Mechanics and Astrodynamics-SIMCA, University of Roma Tor Vergata, University of Milano, Mathematical Physics Italian National Group-GNFM, MIUR grant "Sistemi Dinamici e Applicazioni” (PRIN 2008-2009), European Space Agency—ESA, Telespazio s.p.a.— Roma, Rheinmetall Italia s.p.a., Balletti Park Hotel in San Martino al Cimino-Viterbo (Italy). 